\title{
Highlights from the clinical symposium on Shiga toxin-producing Escherichia coli / haemolytic uremic syndrome, Berlin, September 2011
}

A Jansen (andreas.jansen@ecdc.europa.eu) ${ }^{1}$

1. European Centre for Disease Prevention and Control (ECDC), Stockholm, Sweden

Citation style for this article:

Jansen A. Highlights from the clinical symposium on Shiga toxin-producing Escherichia coli / haemolytic uremic syndrome, Berlin, September 2011

Euro Surveill. 2011;16(39):pii=19977. Available online: http://www.eurosurveillance.org/ViewArticle.aspx?Articleld=19977

Article published on 29 September 2011

On 9 September 2011, the Estrel Convention Center in Berlin was the venue for a first clinical symposium on Shiga toxin-producing Escherichia coli / haemolytic uremic syndrome (STEC/HUS) reflecting on the large STEC outbreak in Germany earlier this year. The German Society of Nephrology (DGfN) invited internationally renowned clinical experts and microbiologists to discuss the basic science and diagnostics of STEC infections and the different options for treating an EHEC-associated HUS, including plasmapheresis, antibody therapy with Eculizumab, and extracorporeal immune adsorption.

Opening the symposium, Helge Karch from Münster University Clinic gave a brief update on the microbiology and the diagnosis of STEC strains. He pointed out that a STEC 0104: $\mathrm{H}_{4}$ strain was first isolated in Germany in 2001 from a child with HUS [1]. This strain (HUSEC041) was not identical with the 2011 outbreak strain, but showed similar molecular features (like the outbreak strain, this isolate was (stx2)-positive and (eae)-negative). A specific multiplex PCR for the 2011 outbreak strain has been developed within three days after the first isolate was available, and was made publicly accessible [2]. Interestingly, the microbiological features of the outbreak strain as known by today could not explain its pathogenicity as observed in the outbreak, leaving several open questions.

Gérard Krause from the Robert Koch-Institute, Berlin, presented the final outbreak report including the epidemiological investigations [3]. So far, there is no evidence that STEC $0104: \mathrm{H}_{4}$ has established as an endemic strain in Germany, but strict surveillance will be continued.

The discrepancy between the (severe) histopathological image of kidney specimens collected from 14 HUS patients and the (relatively positive) long-term disease outcome was highlighted by Udo Helmchen, HamburgEppendorf University Clinic. Intermediate results from 14 HUS cases demonstrated that diffuse glomerular endotheliosis (14/14), and diffuse tubulointerstitial injury (14/14) were the primary histological features of HUS caused by the outbreak strain. Surprisingly, the outcome of these findings was much better than expected. Based on the preliminary results of the follow-up, probably none of the 14 patients will be dependent upon constant dialysis, thus indicating that the initial histopathological ratings were possibly misleading.

The use of Eculizumab, a monoclonal antibody directed against the complement protein $\mathrm{C}_{5}$, in patients with HUS was one of the specific therapeutic challenges of the outbreak. Rolf Stahl, from Hamburg-Eppendorf University Clinic, reflected on his rationale leading to the off-licence use of this treatment in patients not responding to more established treatment options, i.e. plasma exchange therapy. He did not present any results from the analysis of patients' data, though, leaving the question as to whether Eculizumab was effective or not, open.

This outbreak presented a new and surprising clinical picture with regard to the neurological signs of the patients - this was the main point of the presentation from Karin Weissenborn, Hannover Medical School. The neurologist described her experiences with 43 cases which were followed up during their hospital stay, and of which 42 developed HUS. Cognitive dysfunction, including dysphasia $(n=22)$, apraxia $(n=16)$, and agraphia $(n=13)$ were detected among the patients. In addition, panic attacks $(n=13)$ and hyperreflexia $(n=24)$ were common. Seizures/myoclonia occurred in eight patients. The Minimal Mental Status test proved particularly valuable in assessing cognitive dysfunctions in HUS patients. In Magnetic Resonance Imaging (MRI), a bilateral diffusion disorder was observed in the thalamus area and the basal ganglia. However, there were also patients with no MRI findings although they showed severe neurological impairments. Most, but not all cases, showed a complete remission of neurological signs within weeks.

The use of immunoabsorbtion in severe HUS cases was presented by Sylvia Stracke, Greifswald University's 
Medical Faculty. She pointed out that the patients worsened neurologically while on plasma exchange therapy. In addition, the evidence for plasma exchange therapy turned out to be rather weak, with most information derived from uncontrolled case series, and absence of prospective, randomised controlled studies. While searching for a therapeutic alternative (especially for the neurological signs), the characteristic time-span between onset of gastroenteritis and onset of the neurological complications (5-12 days) was the key clinical finding indicating that additional (auto)antibodies were probably involved in the pathogenesis of the severe neurological complications. The intravascular reduction of these (auto)antibodies by immunoabsorbtion was considered to be one possible reason for the success of this therapy with regard to the neurological complications of HUS patients.

With regard to the discussion about the use of antibiotics in EHEC infections, Winfried Kern, from the University of Freiburg, summarised that antibiotics still cannot be generally recommended for EHEC infections. He pointed out that available evidence for or against antibiotic treatment is still sparse. Randomised controlled trials are necessary in order to elucidate whether antibiotics are effective in reducing the morbidity and mortality of EHEC infections. Especially azithromycin and rifaximin should be subject to further clinical studies for the reduction of microbial load in EHEC patients.

Finally, Jan Kielstein, from Hannover Medical School, presented preliminary results from the German EHECHUS registry. So far, data from 589 patients have been included into the registry. Of all HUS patients, $93 \%$ underwent therapeutic plasma exchange, and $36 \%$ received Eculizumab therapy. The overall mortality rate was $4.4 \%$.

To conclude, two major issues of future concern emerged during the symposium: firstly, the lack of evidence with respect to 'established' (or abandoned) therapeutic options in EHEC/HUS; secondly, the uncertainty of prognostic markers - especially with regard to histopathological features of kidney biopsies in HUS patients. The excellent cooperation between different clinicians, and between clinicians and microbiologists, proved to be a valuable resource during the outbreak. However, this outbreak was mainly restricted to Germany. To prepare for a similar event not restricted to a single European country, structures for timely exchange of clinical data and experiences at European level are urgently needed.
3. Robert Koch Institute (RKI). Abschließende Darstellung und Bewertung der epidemiologischen Erkenntnisse im EHEC 0104: $\mathrm{H}_{4}$ Ausbruch Deutschland 2011. [Final presentation and evaluation of the epidemiological evidence in the EHEC 0104: $\mathrm{H}_{4}$ outbreak in Germany 2011]. RKI: 9 Sep 2011. German. Available from: http://www.rki.de/cln_16o/nn_20576o/DE/ Content/InfAZ/E/EHEC/EHEC-Abschlussbericht,templateld=raw , property=publicationFile.pdf/EHEC-Abschlussbericht.pdf

1. Mellmann A, Bielaszewska M, Köck R, Friedrich AW, Fruth A, Middendorf $B$, et al. Analysis of collection of hemolytic uremic syndrome-associated enterohemorrhagic Escherichia coli. Emerg Infect Dis. 2008;14(8):1287-90.

2. University of Münster. Institute for Hygiene. Konsiliarlabor für Hämolytisch- Urämisches Syndrom (HUS). [Consiliar Laboratory for haemolytic uremic syndrome (HUS)]. Münster: Institute for Hygiene. [Accessed 23 Sep 2011]. German. Available from: http://www.ehec.org/ 